\title{
Generation of neuromuscular specificity in Drosophila: novel mechanisms revealed by new technologies
}

\author{
Akinao Nose* \\ Department of Complexity Science and Engineering, Graduate School of Frontier Sciences, University of Tokyo, Kashiwanoha, Kashiwa, Chiba, Japan
}

\section{Edited by:}

Joshua A. Weiner, The University of

lowa, USA

\section{Reviewed by:}

Kai Zinn, California Institute of

Technology, USA

David van Vactor, Harvard Medical

School, USA

*Correspondence:

Akinao Nose, Department of

Complexity Science and

Engineering, Graduate School of

Frontier Sciences, University of

Tokyo, Kashiwanoha 5-1-5, Kashiwa,

Chiba 277-8561, Japan.

e-mail:nose@k.u-tokyo.ac.jp
The Drosophila larval neuromuscular system is one of the best-characterized model systems for axon targeting. In each abdominal hemisegment, only 36 identified motor neurons form synaptic connections with just 30 target muscles in a highly specific and stereotypic manner. Studies in the 1990s identified several cell-surface and secreted proteins that are expressed in specific muscles and contribute to target specificity. Emerging evidence suggests that target selection is determined not only by attraction to the target cells but also by exclusion from non-target cells. Proteins with leucine-rich repeats (LRR proteins) appear to be a major molecular family of proteins responsible for the targeting. While the demonstrated roles of the target-derived cues point to active recognition by presynaptic motor neurons, postsynaptic muscles also reach out and recognize specific motor neurons by sending out cellular protrusions called myopodia. Simultaneous live imaging of myopodia and growth cones has revealed that local and mutual recognition at the tip of myopodia is critical for selective synapse formation. A large number of candidate target cues have been identified on a single muscle, suggesting that target specificity is determined by the partially redundant and combinatorial function of multiple cues. Analyses of the seemingly simple neuromuscular system in Drosophila have revealed an unexpected complexity in the mechanisms of axon targeting.

Keywords: Drosophila, neuromuscular junction, synapse specificity, muscles, motor neurons, target recognition

\section{INTRODUCTION}

After a long journey to the target region, neurons finally gain the blissful opportunity to meet and "mate" with their synaptic partners. In a complex nervous system, however, the neurons still have the daunting task of finding the right partner from among many potential targets in the vicinity. What are the cellular and molecular underpinnings of this romantic yet difficult process? Despite our wealth of knowledge of the molecular mechanisms involved in axon guidance toward the target region (Kolodkin and Tessier-Lavigne, 2011), relatively little is known about the final "mating" of synaptic partners (Sanes and Yamagata, 2009; Shen and Scheiffele, 2010; Maeder and Shen, 2011).

Drosophila neuromuscular connectivity has long been a favorite model system for studying the molecular mechanisms of target selection, as it is possible to apply strong genetics to the formation of highly accessible synaptic connections between the motor neurons and muscles (Keshishian et al., 1996; Rose and Chiba, 2000; Ruiz-Canada and Budnik, 2006). Analyses of this system have pioneered the synaptic targeting studies by identifying specific molecular labels on target cells, such as Connectin (CON), Fasciclin3 (Fas3), and Capricious (Caps), and by showing their roles through genetic analyses in vivo (Nose et al., 1992, 1994, 1997; Chiba et al., 1995; Shishido et al., 1998; Winberg et al., 1998). These early studies provide excellent examples of how cell-to-cell target specificity can be regulated by the function of cell surface and secreted factors expressed on the target cells. In addition, I review findings from more recent studies that utilized technical innovations such as post-genome molecular genetic analyses and high-resolution cellular imaging. I discuss how target specificity is generated in a negative manner by expression of inhibitory cues in non-target cells and how such cues are transcriptionally regulated during development. I also describe how leucine-rich repeat (LRR) proteins have emerged from systematic gain-of-function (GOF) screens as a prominent molecular family that regulates synaptic specificity. Recent live-imaging analyses have also revealed that postsynaptic muscles actively participate in the partner selection.

\section{TARGET RECOGNITION MOLECULES IDENTIFIED IN THE 1990s}

In each hemisegment of Drosophila embryos and larvae, 30 muscle fibers are innervated by 36 motor neurons via one of the six branches of the peripheral nerves: intersegmental nerves (ISN, $\mathrm{ISNb}$, and ISNd), segmental nerves ( $\mathrm{SNa}$ and $\mathrm{SNc}$ ), and a transverse nerve (TN) (Ruiz-Canada and Budnik, 2006). Dye injection to single neurons conducted in the late 1980s first showed that Drosophila motor neurons project to and synapse with specific muscles in a highly reproducible manner (Halpern et al., 1991; Sink and Whitington, 1991a,b). For example, the RP5 motor neuron innervates muscle 12 (M12) and the RP1 motor neurons innervate M13. During development, these motor neurons initially extend a number of filopodia to multiple muscles in the target region, and later restrict the contacts on the target muscle(s) (Sink and Whitington, 1991b). Muscle ablation and 
duplication experiments indicate that each motor neuron selects appropriate targets in an altered cellular environment (Sink and Whitington, 1991c; Chiba et al., 1993). The high degree of precision and the ability of motor neurons to select specific targets in normal and manipulated situations suggest the presence of molecular labels active on individual muscles and prompted the search for such molecules (e.g., Nose et al., 1992; Van Vactor et al., 1993). These studies led to the identification of several candidate target recognition molecules expressed on specific muscle fibers (Figure 1, Table 1; Nose et al., 1992, 1994, 1997; Chiba et al., 1995; Matthes et al., 1995; Rose et al., 1997; Shishido et al., 1998; Winberg et al., 1998).

The LRR proteins CON and Caps, and an immunoglobulin superfamily (IgSF) protein Fas3, are homophilic cell adhesion molecules that are expressed in subsets of muscles and in the motor neurons that innervate these muscles (Figure 1A; Nose et al., 1992, 1994, 1997; Chiba et al., 1995; Kose et al., 1997; Shishido et al., 1998). Ectopic expression of these molecules in muscles dramatically alters target specificity. For example, Caps is expressed in RP5 and its target muscle, M12. When Caps expression is forced in the neighboring non-target M13, Caps-positive RP5 neurons inappropriately connect with M13 in addition to M12 (Figure 2A; Shishido et al., 1998; Taniguchi et al., 2000). Thus, these molecules appear to control target specificity by promoting interaction between specific partner cells. However, only weak phenotypes in the targeting are seen in loss-of-function (LOF) mutants of these molecules, suggesting that their function is redundant (Nose et al., 1994, 1997; Chiba et al., 1995; Shishido et al., 1998; Abrell and Jackle, 2001). In the case of Caps, closely related Tartan (Trn) was later found to be a redundant molecule that contributes to the targeting (Kurusu et al., 2008; see below). Recent studies have shown that Caps also functions

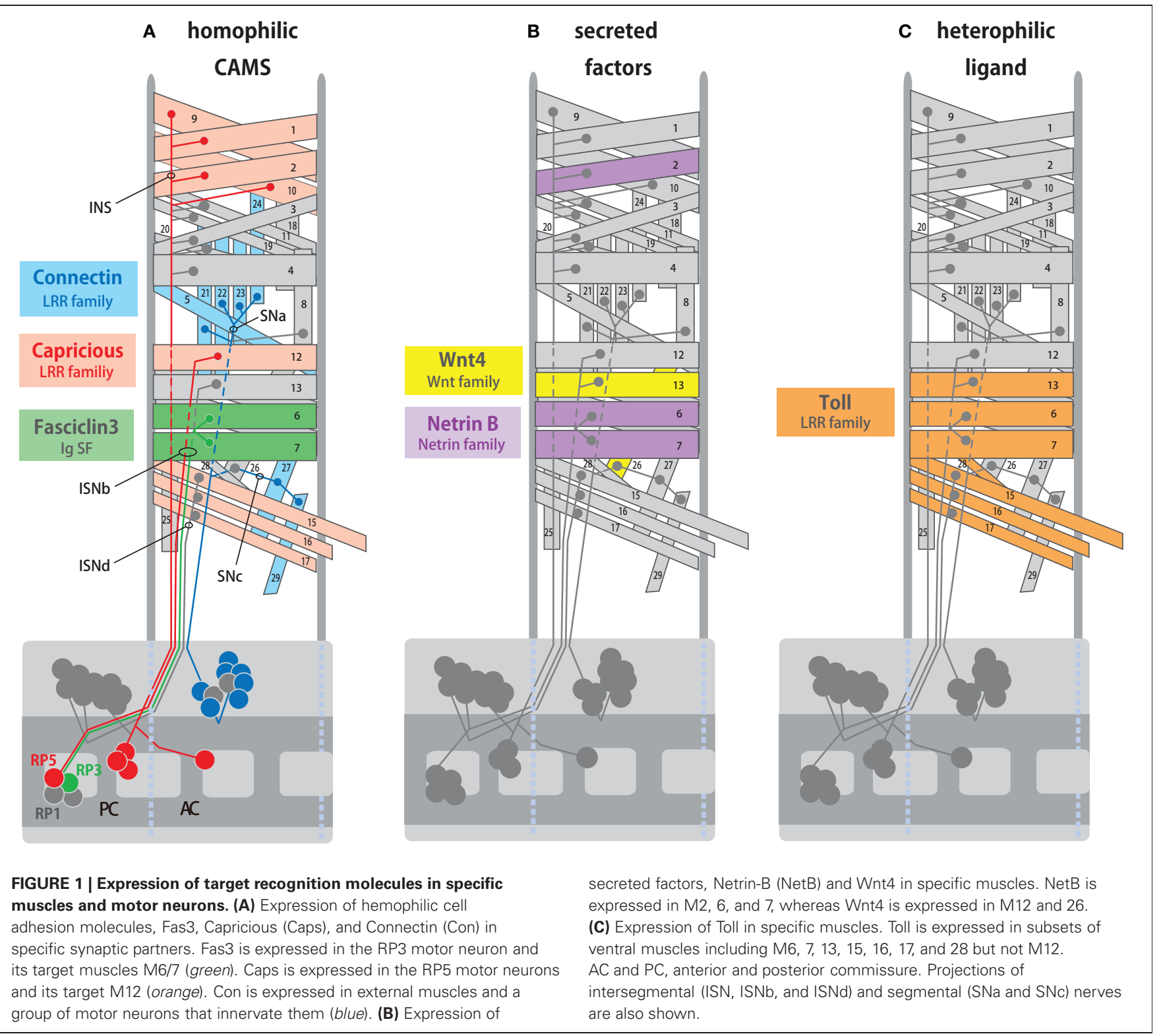


Table 1 | Overview of candidate muscle target recognition cues in Drosophila.

\begin{tabular}{|c|c|c|c|c|c|c|}
\hline Protein & Domain & $\begin{array}{l}\text { Receptors/ } \\
\text { ligands }\end{array}$ & $\begin{array}{l}\text { Expression in } \\
\text { muscles and } \\
\text { motor neurons }\end{array}$ & Phenotypes & $\begin{array}{l}\text { Affected } \\
\text { muscle targets }\end{array}$ & References \\
\hline \multicolumn{7}{|c|}{ CELL ADHESION/RECEPTORS } \\
\hline Capricious (Caps) & LRR & Homophilic & $\begin{array}{l}\text { Subsets of muscles } \\
\text { and motor neurons, } \\
\text { see Figure 1A }\end{array}$ & $\begin{array}{l}\text { LOF and GOF, see } \\
\text { Figure } \mathbf{2 A}\end{array}$ & $\mathrm{M} 12 / 13$ & $\begin{array}{l}\text { Shishido et al. (1998) } \\
\text { and Kurusu et al. } \\
\text { (2008) }\end{array}$ \\
\hline Tartan (Trn) & LRR & $?$ & All ventral muscles & $\begin{array}{l}\text { LOF and GOF, see } \\
\text { Figure } \mathbf{2 A}\end{array}$ & $\mathrm{M} 12 / 13$ & Kurusu et al. (2008) \\
\hline Hattifattener (Haf) & LRR & $?$ & All ventral muscles & $\begin{array}{l}\text { LOF and GOF, see } \\
\text { Figure } \mathbf{2} \mathbf{C}\end{array}$ & $\mathrm{M} 12 / 13$ & Kurusu et al. (2008) \\
\hline $\begin{array}{l}\text { CG8561/convoluted } \\
\text { (Conv) }\end{array}$ & LRR & $?$ & All ventral muscles & $\begin{array}{l}\text { LOF and GOF, see } \\
\text { Figure } \mathbf{2} \mathbf{C}\end{array}$ & $\mathrm{M} 12 / 13$ & Kurusu et al. (2008) \\
\hline Toll & LRR & $?$ & $\begin{array}{l}\text { Subsets of muscles, } \\
\text { see Figure 1C }\end{array}$ & $\begin{array}{l}\text { LOF and GOF, see } \\
\text { Figure } \mathbf{2} \mathbf{B}\end{array}$ & M12/13, M6/7 & $\begin{array}{l}\text { Rose et al. (1997) } \\
\text { and Inaki et al. (2010) }\end{array}$ \\
\hline Connectin (Con) & LRR & Homophilic & $\begin{array}{l}\text { Subsets of muscles } \\
\text { and motor neurons, } \\
\text { see Figure 1A }\end{array}$ & GOF & Lateral muscles & $\begin{array}{l}\text { Nose et al. (1992, } \\
\text { 1994, 1997) }\end{array}$ \\
\hline Fasciclin 3 (Fas3) & $\operatorname{lgSF}$ & Homophilic & $\begin{array}{l}\text { Subsets of muscles } \\
\text { and motor neurons, } \\
\text { see Figure 1A }\end{array}$ & GOF & $\mathrm{M} 6 / 7$ & $\begin{array}{l}\text { Chiba et al. (1995) } \\
\text { and Kose et al. (1997) }\end{array}$ \\
\hline Fasciclin 2 (Fas2) & $\operatorname{lgSF}$ & Homophilic & $\begin{array}{l}\text { All muscles and } \\
\text { motor neurons }\end{array}$ & GOF & All or most muscles & $\begin{array}{l}\text { Davis et al. (1997) } \\
\text { and Winberg et al. } \\
\text { (1998) }\end{array}$ \\
\hline Forked end & - & $?$ & Weak? & LOF and GOF & $\mathrm{M} 12 / 13$ & $\begin{array}{l}\text { Umemiya et al. } \\
\text { (2002) }\end{array}$ \\
\hline \multicolumn{7}{|l|}{ SECRETED FACTORS } \\
\hline Wnt4 & Wnt & Fz-2, Drl-2 & $\begin{array}{l}\text { Subsets of muscles, } \\
\text { see Figure 1B }\end{array}$ & $\begin{array}{l}\text { LOF and GOF, see } \\
\text { Figure } \mathbf{2} \mathbf{B}\end{array}$ & $\mathrm{M} 12 / 13$ & Inaki et al. (2007) \\
\hline Netrin-B (attraction) & Netrin & Fra & $\begin{array}{l}\text { Subsets of muscles, } \\
\text { see Figure 1B }\end{array}$ & LOF and GOF & $\mathrm{M} 6 / 7$ & $\begin{array}{l}\text { Mitchell et al. (1996) } \\
\text { and Winberg et al. } \\
\text { (1998) }\end{array}$ \\
\hline Netrin-B (repulsion) & Ditto & Unc-5 & Ditto & GOF & Lateral muscles & $\begin{array}{l}\text { Winberg et al. (1998) } \\
\text { and Labrador et al. } \\
(2005)\end{array}$ \\
\hline $\begin{array}{l}\text { Semaphorin-2a } \\
\text { (Sema2a) }\end{array}$ & Sema & Plex B & Weak in all muscles & GOF & All or most muscles & $\begin{array}{l}\text { Matthes et al. (1995) } \\
\text { and Winberg et al. } \\
\text { (1998) }\end{array}$ \\
\hline Beat-IIIc & $\operatorname{lgSF}$ & $?$ & $\begin{array}{l}\text { Subsets of muscles } \\
(13 \text { and } 30)\end{array}$ & GOF & $\mathrm{M} 12 / 13$ & Inaki et al. (2007) \\
\hline Sulfated (Sulf1) & Sulfatase & $?$ & $\begin{array}{l}\text { Large subsets } \\
\text { (higher in M13 than } \\
\text { in } \mathrm{M} 12 \text { ) }\end{array}$ & GOF & $\mathrm{M} 12 / 13$ & Inaki et al. (2007) \\
\hline CG6867 & $\operatorname{lgSF}$ & $?$ & $\begin{array}{l}\text { Most or all muscles } \\
\text { (higher in M13 than } \\
\text { in } M 12 \text { ) }\end{array}$ & GOF & $\mathrm{M} 12 / 13$ & Inaki et al. (2007) \\
\hline Glutactin (GIt) & Chorinesterase & $?$ & $\begin{array}{l}\text { Weak (higher in } \mathrm{M} 13 \\
\text { than in } \mathrm{M} 12 \text { ) }\end{array}$ & GOF & $\mathrm{M} 12 / 13$ & Inaki et al. (2007) \\
\hline $\begin{array}{l}\text { Larval serum protein } \\
2 \text { (Lsp2) }\end{array}$ & - & $?$ & $\begin{array}{l}\text { Weak (higher in } \mathrm{M} 13 \\
\text { than in } \mathrm{M} 12 \text { ) }\end{array}$ & GOF & $\mathrm{M} 12 / 13$ & Inaki et al. (2007) \\
\hline
\end{tabular}

Attractive and inhibitory cues are shown in red and blue color, respectively. 


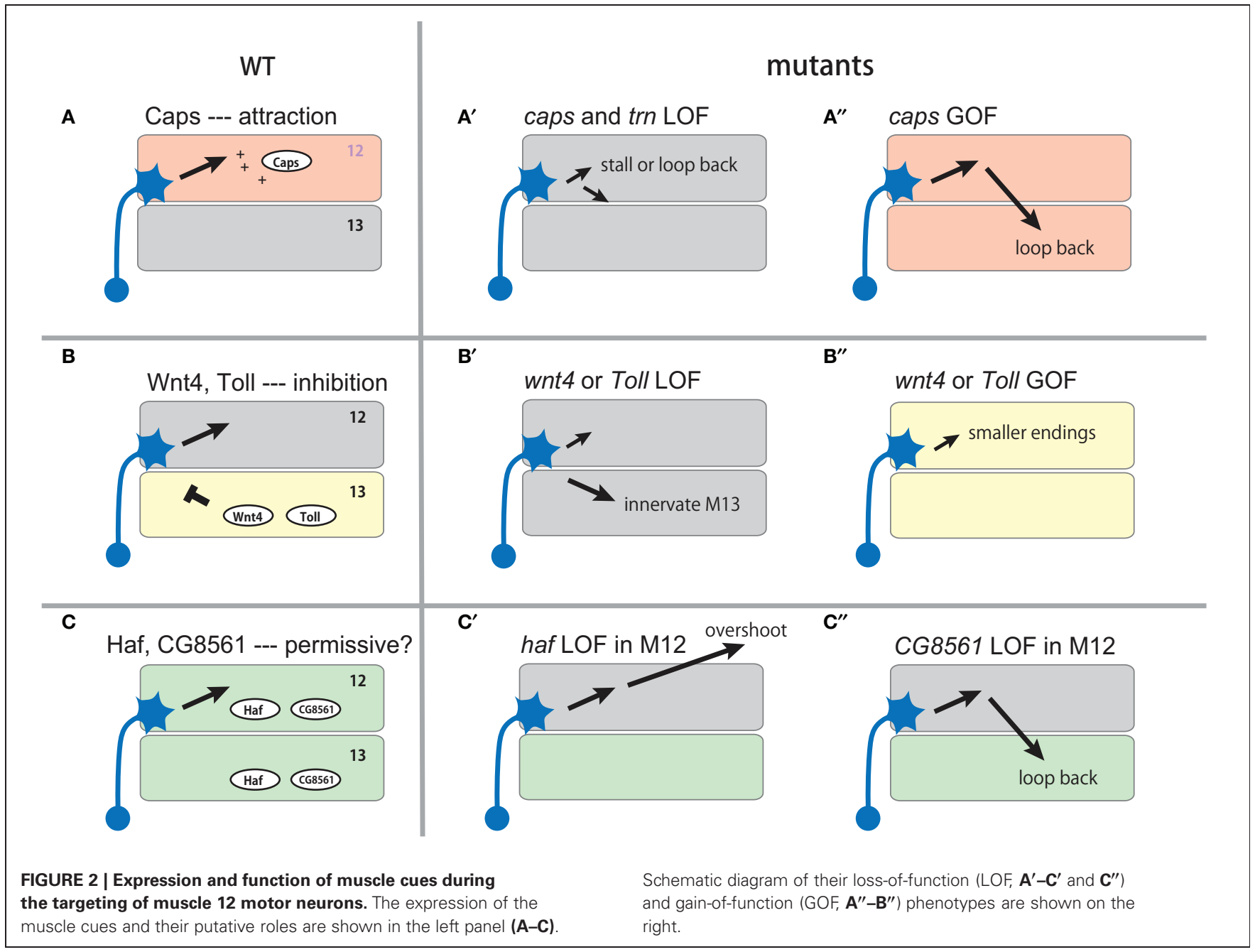

as a target recognition molecule in the visual system and olfactory system. In the visual system, as in the neuromuscular system, Caps is expressed in specific synaptic partners: the presynaptic photoreceptor R8 and the target layer M3 in the medulla. LOF and GOF analyses of Caps support the notion that Caps mediates specific interaction between R8 photoreceptors and the M3 layer (Shinza-Kameda et al., 2006). Several other cell-surface molecules have also been implicated in the targeting of the photoreceptors, including N-cadherin, Flamingo, DLAR, and Golden Goal (Hadjieconomou et al., 2011), although their roles in neuromuscular targeting remains unknown. In the olfactory system, Caps has been implicated in the targeting of projection neuron dendrites to specific glomeruli (Hong et al., 2009). In this case, heterophilic interaction with unknown ligand(s) is proposed as a mechanism because the target cells do not express Caps.

Netrin-B (NetB), a secreted protein of the Netrin family, is expressed in subsets of muscles, including M6/7 innervated by RP3 (Figure 1B, Mitchell et al., 1996; Winberg et al., 1998). In the absence of NetB, RP3 neurons form reduced endings on the target muscles, suggesting that NetB functions as an attractive cue for the neurons. NetB also repels other neurons ( $\mathrm{SNa}$ and
SNc motor neurons), when it is ectopically expressed in all muscles (Winberg et al., 1998). Frazzled (Fra) and Unc5 receptors are implicated as receptors for attractive and repulsive signaling by NetB, respectively (Kolodziej et al., 1996; Mitchell et al., 1996; Keleman and Dickson, 2001; Labrador et al., 2005).

Another molecule, Toll, has been shown to inhibit motor innervation when overexpressed in muscles. Toll is an LRR cell surface protein and is expressed in subsets of ventral muscles (Figure 1C; Nose et al., 1992; Halfon et al., 1995). Toll has antisynaptogenic effects on RP3 (Rose et al., 1997). RP3 fails to innervate the target muscles, M6/7, when Toll is overexpressed in these muscles. In Toll LOF mutants, RP3 shows a variety of axon projection and targeting defects. Toll is also expressed in $\mathrm{M} 6 / 7$, but the expression is diminished by the time RP3 arrives. Thus, temporal and spatial regulation of Toll has been proposed to be critical for RP3 targeting (Rose et al., 1997). Studies of NetB and Toll show that ectopic expression of inhibitory cues can change neuromuscular targeting. However, it is unclear whether these molecules are essential for target selection.

Target specificity can also be changed by altered expression of uniform cues expressed in all muscles. Fasciclin2 (Fas2) is a homophilic cell adhesion molecule of IgSF and is expressed in 
all motor neurons and muscles. Although Fas2 is not required for formation of neuromuscular specificity, changing the relative level of Fas2 in neighboring muscles dramatically changes the pattern of target selection (Davis et al., 1997). Sema-2a (previously termed SemaII) is a secreted protein of the semaphorin family and is weakly expressed in all muscles in the body wall (it is also strongly expressed in a single ventral muscle specific to thoracic segment T3; Matthes et al., 1995). Like Fas2, Sema-2a is not essential for neuromuscular targeting. However, overexpression of Sema-2a in muscles inhibits synapse formation by many motor neurons (Matthes et al., 1995; Winberg et al., 1998).

Several lines of evidence suggest that individual muscles are not specified in a lock-and-key fashion by unique molecular labels for targeting. First, motor neurons innervate non-target muscles when the target cell(s) are absent (Cash et al., 1992). Second, when a muscle fails to be innervated by the normal partners, other motor neurons inappropriately target the muscle (Halfon et al., 1995; Kopczynski et al., 1996). Third, LOF of any of the identified candidate target recognition molecules only partly disrupts proper targeting (Nose et al., 1994; Chiba et al., 1995; Matthes et al., 1995; Shishido et al., 1998; Winberg et al., 1998). It is, therefore, generally believed that target specificity is determined by the combined function of multiple cues in a dynamic and flexible manner (relative balance model; Winberg et al., 1998). The cues could be uniquely expressed on specific muscles or general cues expressed in all muscles. Synaptic connections are also refined in an activity-dependent manner once they are formed (Carrillo et al., 2010). Thus, despite the stereotypy during normal development, the neuromuscular connectivity appears to be a flexible and plastic system.

Winberg et al. (1998) and Rose and Chiba (1999) studied whether motor neurons can indeed integrate information provided by multiple cues by simultaneously changing the expression of multiple cues. Winberg et al. (1998) systematically altered the expression of $\mathrm{NetB}$, an attractive cue, and the inhibitory cue Sema-2a during the targeting of $\mathrm{M} 6 / 7$ by RP3. As described above, removal of attractive NetB reduces innervation of M6/7 by RP3 as does increased expression of inhibitory Sema-2a. However, when the removal of NetB is combined with the removal of Sema-2a, or when the increased expression of Sema- $2 a$ is combined with increased expression of $\mathrm{NetB}$, the defects in targeting were restored. Similarly, Rose and Chiba (1999) simultaneously manipulated the expression of Fas3, an attractive cue for RP3, and Toll, an inhibitory cue, and found that they cancelled one other. These observations strongly support the idea that motor neurons can assess the balance between multiple attractive and inhibitory cues during targeting.

\section{NEW MOLECULAR AND GENETIC SCREENS IN THE POST-GENOME ERA}

The studies described above strongly suggest that target specificity of individual muscles is determined by the action of multiple target cues. However, the following questions remain. How many molecules are involved in the specification of individual target muscles? Is the specificity generated by diversity among a particular protein family or by random utilization of different protein families? What are the roles of inhibitory cues in target selection?
How is the expression of multiple target cues in specific muscles coordinated during development? To answer these questions, two recent studies utilized high-throughput, post-genomic technologies to search for genes involved in the targeting (Inaki et al., 2007; Kurusu et al., 2008). Both of these studies focused on the targeting between M12 and M13 and tried to systematically identify genes that regulate synaptic specificity. M12 is innervated by RP5 and V neurons (collectively called MN12s) and M13 is innervated by RP1 and RP4. Inaki et al. (2007) performed comparative microarray analyses of $\mathrm{M} 12$ and M13 by directly isolating these muscles from dissected embryos. The analyses identified more than 200 candidate genes that are differentially expressed between these muscles, including 25 that encodes putative cell surface and secreted proteins that could play roles in target recognition. Functional analyses of the candidate genes led to the identification of a negative regulator of target specificity, Wnt4, and a transcriptional regulator of target specificity, Tey, as detailed below. Kurusu et al. (2008) conducted systematic GOF analyses of a collection of putative cell surface and secreted proteins identified by bioinformatics. The screening revealed roles for a number of LRR proteins, highlighting the roles of this gene family in selective synapse formation.

\section{GENERATION OF TARGET SPECIFICITY BY NEGATIVE CUES}

Microarray analyses by Inaki et al. (2007) identified Wnt4, a secreted Wnt family protein, as being enriched in M13. In Wnt4 LOF mutants, MN12s form smaller synapses on their normal target and form ectopic nerve endings on M13. This results in enlarged nerve terminals on M13 and diminished endings on M12. Conversely, when Wnt4 is ectopically expressed on M12, the nerve terminal on the muscle is greatly reduced in size. These results suggest that Wnt4 expressed on M13 prevents synapse formation by the MN12s (Figure 2B). Frizzled-2 (Fz-2) and Derailed-2 (Drl-2) receptors and a downstream Dishevelled (Dsh) have been implicated in the reception of Wnt4 signaling in motor neurons. Whereas Fz-2 is expressed in all neurons, drl-2 expression is restricted to specific neurons and thus may be responsible for the specificity of the Wnt 4 action. Studies on synapse formation in C. elegans showed that the Wnt protein Lin-44 determines the subcellular location of synapses by preventing synapse formation in a specific domain of DA9 axons (Klassen and Shen, 2007). Thus, regulation of synaptic specificity by inhibitory activity of Wnts appears to be an evolutionarily conserved mechanism.

Another candidate target recognition molecule on M13 that has been identified by microarray screening is Toll. As described above, Toll was previously implicated as an inhibitory cue expressed on M6/7 (Rose et al., 1997). The differential expression of Toll in M12 and M13 prompted Inaki et al. (2010) to study potential roles of Toll in target selection between these two muscles. The authors found that Toll mutant embryos show similar phenotypes as seen in Wnt4 mutants: expansion of M13 terminals and reduction of M12 terminals. Ectopic expression of Toll in M13 also showed similar phenotypes as seen when Wnt4 is misexpressed on the muscle: reduction of M12 terminals. These results suggest that Toll, like Wnt4, regulates target specificity by inhibiting synapse formation on M13 (Figure 2B). How Toll transmits 
the inhibitory signal to motor neurons remains unknown. One possibility is that Toll functions as a ligand expressed on muscles and signals through unknown receptor(s) on motor neurons. Toll is the founding member of the Toll-like receptors, whose functions as receptors have been well characterized in early embryogenesis and innate immunity (West et al., 2006; Valanne et al., 2011). Whether or not Toll also functions as a ligand for other receptors is currently unknown.

It is notable that M12MNs form nerve endings of similar size on M12 and M13 in mutants that lack Wnt4 or Toll. This indicates that in the absence of inhibitory cues, these two muscles are equally favorable targets for the motor neurons. Target specificity only arises with the differential expression of these negative cues. These findings provide strong evidence for the role of inhibition in target selection.

In addition to Wnt 4 and Toll, microarray analyses by Inaki et al. (2007) identified five molecules that are enriched in M13 and, when misexpressed in M12, inhibit synapse formation of MN12s: Beaten path III-c (Beat-IIIc, a member of the Beat subfamily of IgSF), Glutactin (Glt) (a cell surface protein with cholinesterase domains), Larval serum protein 2 (Lsp2) (a secreted protein), Sulfated (Sulf1) (an extracellular sulfatase), and CG6867 (a secreted protein of IgSF) (Table 1). Although the precise roles of these molecules remain to be determined by LOF analyses, these results suggest that a single muscle expresses a variety of inhibitory cues. Beat-IIIc is of particular interest since a recent study showed that another member of the family, Beat-Ia, and its ligand, Sidestep (Side), are involved in the guidance of motor axon to the target region (Siebert et al., 2009). Identification of Beat-IIIc as a putative muscle target recognition molecule suggests a possibility that Beat/Side families may be important regulators of synaptic targeting.

\section{LRR PROTEIN FAMILY AS KEY REGULATORS OF SELECTIVE SYNAPSE FORMATION}

Based on the fact that GOF mutants of many identified target recognition molecules show stronger phenotypes than their LOF counterparts. Kurusu et al. (2008) performed systematic GOF screening in search of new target cues. The authors screened $\sim 410$ putative cell surface or secreted proteins (representing $\sim 40 \%$ of this class of protein in Drosophila) and found that targeted expression of 30 of these genes in all muscles produces mistargeting phenotypes. Eleven of these genes (including five known genes) are normally expressed in muscles. Remarkably, five of the 11 genes are LRR proteins, while all other genes contained different domains. Two of the LRR proteins are Caps and its close relative Tartan. The identification of the two closely related genes by the screening prompted the authors to study caps, trn double mutants. Single mutants of trn or caps display only weak phenotypes in ISNb guidance and targeting. However, in the double mutants, a dramatic phenotype called "terminal loop" was observed in ISNb targeting. The loop is seen on the distal end of the ISNb near the M12/M13 boundary or on M12, suggesting that M12MNs either stall or turn back toward M13 after reaching the target (Figure 2A). Caps and Trn thus appear to function in a redundant manner in M12 targeting. Whereas caps is enriched in M12 and other specific muscles, trn appears to be expressed weakly in all ventral muscles with a lower level in M12 and M13 compared to M6 and M7 (Kurusu et al., 2008).

The GOF screening also identified two novel LRR cell surface proteins, Hattifattener (Haf) and CG8561/Convoluted (Conv), as regulators of ISNb targeting. Since expression of these genes is not confined to specific muscles, they appear to function as general muscle cues that are essential for proper ISNb targeting. In haf mutant embryos, ISNb motor neurons display a variety of abnormal projection and targeting errors, which are hard to interpret in terms of simple mechanisms. However, a more informative phenotype was observed when the gene was knocked down specifically in M12 by expression of RNAi constructs (Figure 2C). In the M12-specific mutants, the distal branch of ISNb reaches and innervates M12 as in normal animals, but it also extends further and forms ectopic synapses with lateral muscles. This phenotype suggests a role for haf in stabilizing the connection with the target muscles. Another LRR protein, CG8561/Conv is an ortholog of vertebrate acid-labile subunit (Als) of the IGF1 binding complex. Like haf, LOF of this gene in all muscles produces complex phenotypes. However, when the function of CG8561 was knocked down only in M12, a specific loop-back phenotype of M12MNs was seen in $>70 \%$ of the segments (Figure 2C).

In addition to genes implicated in targeting, the screening by Kurusu et al. also identified a number of genes whose forced expression in muscles affects neuromuscular junction (NMJ) morphology. In total, 53 LRR genes (of the 101 LRR genes in the genome) were screened and 16 of them were found to affect target selection, synapse formation or both. In addition, previous studies have already identified three LRR proteins (Caps, CON, and Toll) as target cues (Table 1). Thus, members of this family appear to be major players in selective synapse formation in this system (Table 1). LRRs are repeating motifs of $\sim 24$ amino acids that are thought to function as an effective and versatile protein-binding motif (de Wit et al., 2011). Expression of diverse members of this family could, therefore, generate specificity among neurons by conferring differential adhesion and/or recognition activity. Recent studies on mammalian synapse formation also identified a number of LRR transmembrane proteins as synaptic organizers (e.g., Linhoff et al., 2009; de Wit et al., 2011). Some of these molecules are expressed in a region-specific manner. While their roles in the control of synaptic specificity remain to be explored, it is an interesting possibility that LRR proteins also control synaptic specificity in the vertebrate brain.

\section{TRANSCRIPTIONAL CONTROL OF TARGET SPECIFICITY}

The demonstrated roles of multiple muscle target cues raise a further question: what are the higher-level processes that regulate the expression of these cues? For proper neural wiring, target recognition cues and their receptors have to be expressed in specific pre- and post-synaptic cells with proper timing. Little is known about the developmental mechanisms that regulate this process. A study by Inaki et al. (2010) provides an important insight. One of the genes identified by microarray screening (Inaki et al., 2007) encodes the putative transcription factor Tey. Its expression in 
muscles is highly specific, being confined to a single muscle, M12, among the 30 muscles in the body wall. Tey represses the expression of Toll in this muscle. As described above, Toll is an inhibitory cue for motor neurons targeted to M12 and is expressed in M13 and other surrounding non-target muscles, but not in M12. In tey LOF mutants, Toll is ectopically expressed in M12. Conversely, ectopic expression of tey in M13 downregulates Toll expression in the muscle. LOF and GOF of tey lead to phenotypes as expected for the misregulated expression of Toll. Synapse formation on M12 is reduced in tey LOF mutants, consistent with the upregulation of Toll in this muscle. M13 receives ectopic innervation in tey GOF mutants, consistent with the downregulation of Toll. Tey, therefore, controls neuromuscular specificity by repressing the expression of Toll in M12 (Figure 3A). This example shows how target specificity can be generated by suppression of an inhibitory cue in one among a group of target cells expressing the cue (Figure 3B).

Control of target specificity by transcriptional repression is also seen in presynaptic photoreceptor cells in the Drosophila visual system (Morey et al., 2008). As described above, Caps is expressed in R8 photoreceptor cells and is required for their proper projection to the target layer. Caps expression in R8 cells is positively regulated by the transcription factor Senseless (Sens). In R7 cells, on the other hand, another transcription factor NF-YC represses expression of Sens and the downstream Caps. In NF-YC mutants, R7 cells misexpress Sens and Caps and inappropriately terminate in the R8 target layer as seen when Caps is ectopically expressed in these cells. Thus, repression of Sens and Caps is critical for R7 targeting.

In tey mutants, muscle insertion sites of M12 are also specifically affected. Thus, tey appears to regulate two key aspects of muscle specification: geometry and synaptic specificity. Several transcription factors have been identified that are expressed in subsets of muscle and/or their progenitor cells (muscle founder cells) and specify the identities of the muscles (reviewed in Tixier et al., 2010). It is an interesting possibility that these genes, like tey, may also regulate neuromuscular target specificity. A BTB zincfinger transcription factor, Abrupt $(\mathrm{Ab})$, on the other hand, is expressed in all muscles but regulates specific aspects of motor neuron projection (Hu et al., 1995). In abrupt mutants, ISNb motor neurons reach the target region normally but fail to form appropriate innervation on the target muscles. The differentiation of the target ventral muscles is normal. Ab may thus regulate the expression of general target recognition molecules, such as Haf, in these muscles. Several transcription factors that function in motor neurons to specify the target domain have also been identified, including Even-skipped, Islet and Lim3 (reviewed in Landgraf and Thor, 2006). However, whether these transcription factors regulate the expression of target recognition molecules remain unknown.

\section{PARTNER RECOGNITION BY MYOPODIA}

In addition to recognition by presynaptic motor neurons, recent studies suggest that the postsynaptic muscles also seek and find their presynaptic partners. In fixed specimens, differentiated muscles show little sign of motility and, therefore, have long been regarded as passive players during synaptic matchmaking, waiting

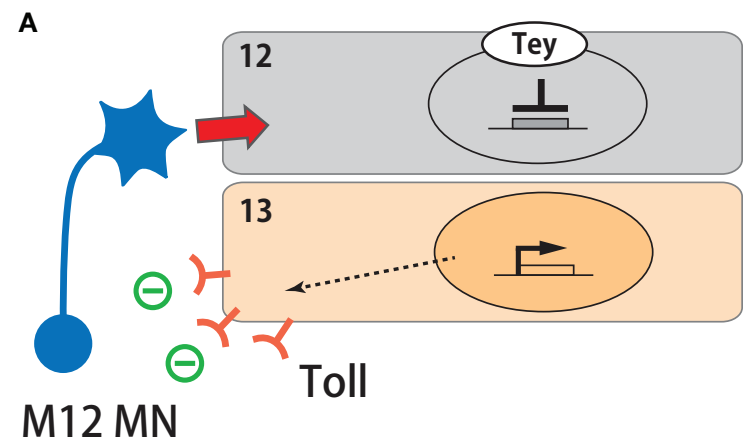

B

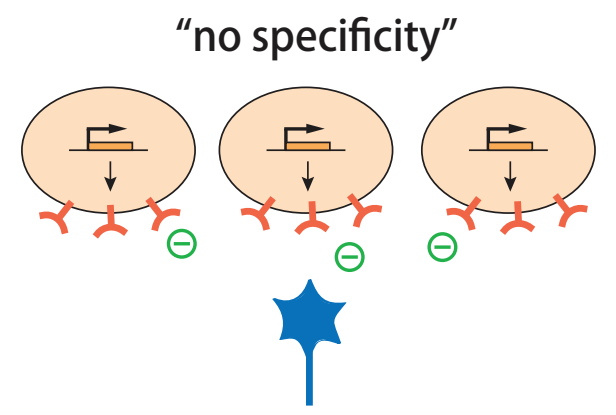

"Specificity"
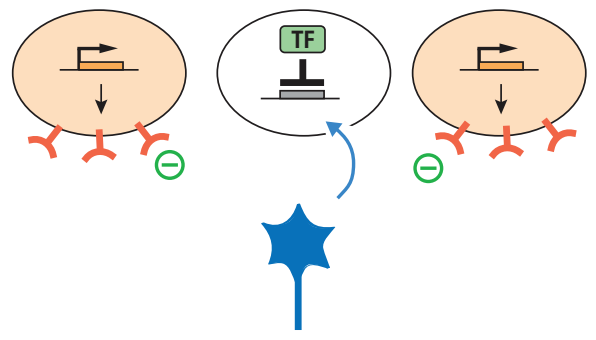

FIGURE 3 | Transcriptional regulation of target specificity by repression of inhibitory cues. (A) Tey represses expression of Toll in M12 and thereby determines target specificity. (B) A model in which target specificity can be generated in a group of equivalent targets by transcriptional repression of a repulsive cue.

for the arrival of motor neurons. However, Ritzenthaler et al. (2000) used high-resolution live imaging to show that muscles actually bear a number of dynamic protrusions, called myopodia, that extend and retract prior to and during the period of neuromuscular interaction. Myopodia are actin-based membrane microprocesses $\sim 10 \mu \mathrm{m}$ or longer in length and $0.1-0.2 \mu \mathrm{m}$ in diameter. Myopodia initially extend in random directions but gradually cluster at the site of innervation as they interact with the presynaptic filopodia (Figure 4A, top). The clustering of myopodia depends on interaction with the presynaptic motor neurons (Ritzenthaler et al., 2000; Ritzenthaler and Chiba, 2003). 
A myopodia clustering and formation of the nascent synaptic site
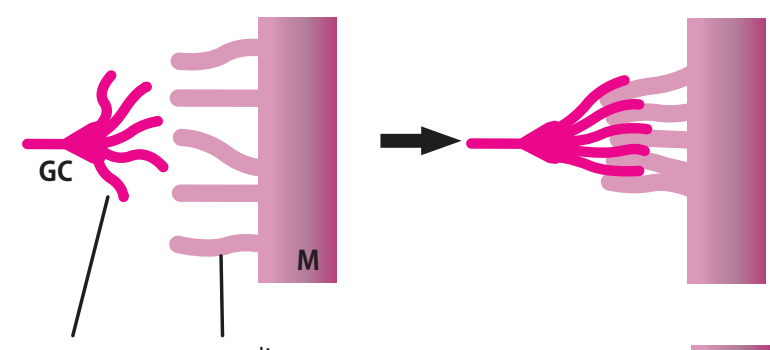

myopodia

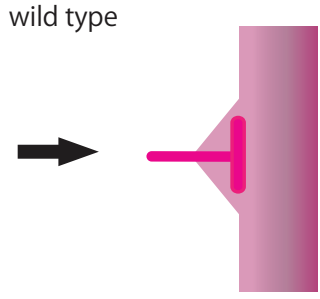

caps, trn mutants

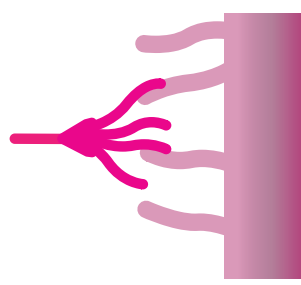

c Caps accumulation at the tips of myopodia

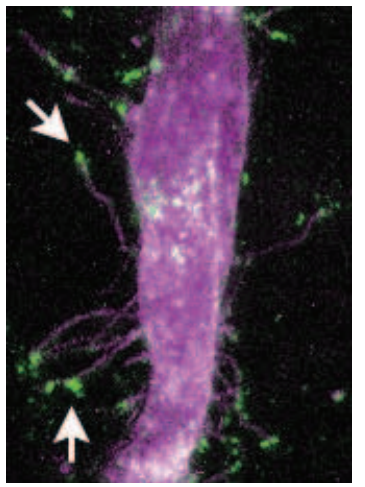

B Partner selection by the postsynaptic myopodia

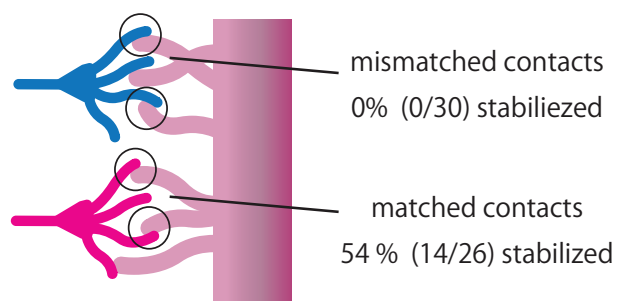

FIGURE 4 | Partner recognition by myopodia. (A) Schematic diagram showing myopodia clustering and formation of nascent synaptic sites in wild type (above) and caps, trn double mutants (below). (B) Partner selection by myopodia (Left). While $54 \%$ of myopodial contacts with a partner motor neuron (magenta) are stabilized, none of the contacts with the non-partner motor neuron (b/ue) are stabilized (Right). Caps at the tip of myopodia may
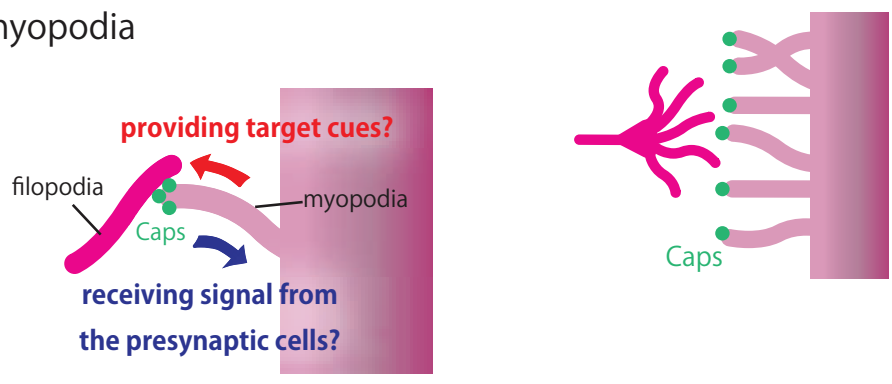

function in a bidirectional manner, providing target cues to and receiving signal from the presynaptic cells. (C) Caps accumulation at the tips of mypopodia. GC, growth cone (Top). Simultaneous live-imaging of Caps-GFP (green) and muscle membrane (magenta) reveals Caps localization at the tips of myopodia (arrows). Taken from Kohsaka and Nose (2009) Bottom. A diagram showing Caps localization.
As myopodia cluster, they intermingle and "zip" with the presynaptic filopodia and finally fuse to form the nascent postsynaptic sites (Ritzenthaler et al., 2000; Ritzenthaler and Chiba, 2003; Kohsaka et al., 2007). Thus, neuromuscular targeting is a reciprocal process in which motor neurons and muscles seek each other.

What is the precise role of myopodia? Cells use filopodia and other cellular extensions to efficiently transmit and receive information via long-distance direct cell-cell interaction (Rørth, 2003; Hsiung et al., 2005; Heiman and Shaham, 2010; Roy et al., 2011). For example, neuronal growth cones extend filopodia as they explore the local environment. In the Drosophila imaginal disc, cytonemes and other actin-based extensions are utilized not only in the signal-receiving cells to actively seek cues from a distance but also in the signal-sending cells to efficiently present information to the receiving cells (Rørth, 2003; Hsiung et al., 2005; Roy et al., 2011). Similarly, myopodia may function to receive and/or send information at a distance. A study by Kohsaka and
Nose (2009) showed that myopodia have a sensory function. Simultaneous live-imaging of muscles and motor axons is used to study the behavior of myopodia when they encounter partner or non-partner motor neurons (Figure 4B). When myopodia encounter the growth cones of partner motor neurons, about half of the contacts are stabilized to from synapses. In contrast, none of the contacts with the non-partners are stabilized, suggesting that individual myopodia can recognize the appropriate partner. So, just like presynaptic growth cones seek and find the right targets, postsynaptic muscles also select among potential presynaptic partners using myopodia.

Localization and function of Caps in myopodia has also been studied (Kohsaka and Nose, 2009). Caps is localized at the tip of myopodia, where many of the initial contacts between muscles and growth cones take place (Figure 4C). In caps mutants, fewer myopodial contacts with the presynaptic motor neurons are stabilized in M12. The size of the nascent presynaptic terminal is also reduced. Similar but more penetrant phenotypes are 
seen in caps, trn double mutants (Figure 4A, bottom). Thus, Caps-mediated signaling at the tip of myopodia appears to be critical for selective synapse formation. It is likely that Caps functions in a bi-directional manner at the tips of myopodia (Figure 4B, right). By localizing at the tip of myopodia, Caps may be efficiently presented to and recognized by the growth cones. Caps may also be involved in the recognition of motor neurons by the myopodia. Such bidirectional signaling might ensure proper synaptic matchmaking. Many signaling and adhesion molecules are known to accumulate at the tips of filopodia, including Ena/VASP, formins, and integrins (Faix and Rottner, 2006; Mattila and Lappalainen, 2008). Thus, tips of myopodia may function as a signaling center that regulate myopodia dynamics and synapse formation. Dendrites of vertebrate neurons also cast out dendritic filopodia during synapse formation. A recent study in rodents showed that dendritic filopodia of hippocampal neurons discriminate between target and non-target axons (Lohmann and Bonhoeffer, 2008). Regulation of synapse specificity by postsynaptic protrusions thus seems to be a common mechanism across taxa.

\section{CONCLUSION}

When investigators began searching for NMJ target recognition molecules in the 1990s, most envisioned a simple lock-and-key mechanism, as initially postulated by Sperry (1963). However, the dance between motor neurons and target muscles turns out to be far more complex than initially thought. Multiple molecular chaperons appear to mediate the partner selection in a partially redundant and combinatorial manner. Partner selection is not only determined by attraction to the partner but also by exclusion from other cells. Furthermore, the molecular courtship is not one-sided but is a mutual searching process mediated by complex interactions of filopodia and myopodia.

Studies thus far implicate at least 11 proteins in targeting of M12, including the attractive cues Caps and Tartan on the muscle, inhibitory cues Wnt4, Toll, Beat-3c, Glt, Lsp2, Sulf1, and CG6867 on the neighboring M13, and general cues Haf and CG8561 on all muscles. Why so many target cues are present to specify the targeting of a single muscle is a mystery. Determining how multiple

\section{REFERENCES}

Abrell, S., and Jackle, H. (2001). Axon guidance of Drosophila $\mathrm{SNb}$ motoneurons depends on the cooperative action of muscular Kruppel and neuronal capricious activities. Mech. Dev. 109, 3-12.

Carrillo, R. A., Olsen, D. P., Yoon, K. S., and Keshishian, H. (2010). Presynaptic activity and CaMKII modulate retrograde semaphorin signaling and synaptic refinement. Neuron 68, 32-44.

Cash, S., Chiba, A., and Keshishian, H. (1992). Alternate neuromuscular target selection following the loss of single muscle-fibers in Drosophila. J. Neurosci. 12, 2051-2064.
Chiba, A., Hing, H., Cash, S., and Keshishian, H. (1993). Growth cone choices of Drosophila motoneurons in response to muscle-fiber mismatch. J. Neurosci. 13, 714-732.

Chiba, A., Snow, P., Keshishian, H., and Hotta, Y. (1995). FasciclinIII as a synaptic target recognition molecule in Drosophila. Nature 374, 166-168.

Davis, G. W., Schuster, C. M., and Goodman, C. S. (1997). Genetic analysis of the mechanisms controlling target selection: target-derived fasciclin II regulates the pattern of synapse formation. Neuron 19, 561-573.

de Wit, J., Hong, W., Luo, L., and Ghosh, A. (2011). Role of

classes of molecular cue are translated into a coherent downstream signal that culminates in the formation of specific synapses is an important future direction. Development of a complete set of GOF constructs for all cell surface and secreted proteins would allow the definition of every protein that can produce a neuromuscular targeting GOF phenotype and provide a list of candidate players. Even more mysterious is how the expression of diverse target cues is coordinated during muscle development. Although studies of Tey begin to answer this question, much more work is needed to obtain the whole picture. Clues may be obtained by comprehensive analyses of the transcriptional cascade, like those performed in the Drosophila mesoderm (Zinzen et al., 2009; Junion et al., 2012).

\section{NOTE ADDED IN REVISION}

Mosca et al. (2012) recently reported that Teneurin (Ten)-m and Ten-a, belonging to conserved epidermal growth factor (EGF)repeat-containing transmembrane proteins, regulate neuromuscular targeting in Drosophila. Ten-m and Ten-a are expressed in all muscles and motor neurons, respectively, and mediate bidirectional interaction required for proper organization of the NMJs. Ten-m is expressed at a higher level in muscle 3 and 8 , and are required for synapse formation in these muscles. Like Fas2, changing the relative level of Ten-m between muscles 6 and 7 alters the pattern of target selection. Thus, Ten-m and Ten-a appear to regulate general synaptic organization as well as target specificity.

\section{ACKNOWLEDGMENTS}

I thank present and past members of the lab for their contribution to the studies described in this article and Kasumi Shibahara for the help in illustration. This article is supported by a Grantin-Aid for Scientific Research on Innovative Areas "Mesoscopic Neurocircuitry” (No. 22115002) and for Scientific Research on Innovative Areas "Comprehensive Brain Science Network" (No. 221S0003), of The Ministry of Education, Culture, Sports, Science, and Technology, Japan, and for Scientific Research (B) (No. 23300114) of Japan Society for the Promotion of Science (JSPS), to Akinao Nose.

leucine-rich repeat proteins in the development and function of neural circuits. Annu. Rev. Cell Dev. Biol. 27, 697-729.

Faix, J., and Rottner, K. (2006). The making of filopodia. Curr. Opin. Cell Biol. 18, 18-25.

Hadjieconomou, D., Timofeev, K., and Salecker, I. (2011). A step-by-step guide to visual circuit assembly in Drosophila. Curr. Opin. Neurobiol. 21, 76-84.

Halfon, M. S., Hashimoto, C., and Keshishian, H. (1995). The Drosophila Toll gene functions zygotically and is necessary for proper motoneuron and muscle development. Dev. Biol. 169, 151-167.
Halpern, M. E., Chiba, A., Johansen, J., and Keshishian, H. (1991). Growth cone behavior underlying the development of stereotypic synaptic connections in Drosophila embryos. J. Neurosci. 11, 3227-3238.

Heiman, M. G., and Shaham, S. (2010). Twigs into branches: how a filopodium becomes a dendrite. Curr. Opin. Neurobiol. 20, 86-91.

Hong, W., Zhu, H., Potter, C. J., Barsh, G., Kurusu, M., Zinn, K., and Luo, L. (2009). Leucine-rich repeat transmembrane proteins instruct discrete dendrite targeting in an olfactory map. Nat. Neurosci. 12, 1542-1550.

Hsiung, F., Ramirez-Weber, F. A., Iwaki, D. D., and Kornberg, T. B. (2005). Dependence of Drosophila 
wing imaginal disc cytonemes on Decapentaplegic. Nature 437, 560-563.

Hu, S., Fambrough, D., Atashi, J., Goodman, C., and Crews, S. (1995). The Drosophila abrupt gene encodes a BTB-zinc finger regulatory protein that controls the specificity of neuromuscular connections. Genes Dev. 9, 2936-2948

Inaki, M., Shinza-Kameda, M., Ismat, A., Frasch, M., and Nose, A. (2010). Drosophila Tey represses transcription of the repulsive cue Toll and generates neuromuscular target specificity. Development 137, 2139-2146.

Inaki, M., Yoshikawa, S., Thomas, J. B., Aburatani, H., and Nose, A. (2007). Wnt4 is a local repulsive cue that determines synaptic target specificity. Curr. Biol. 17, 1574-1579.

Junion, G., Spivakov, M., Girardot, C., Braun, M., Gustafson, E. H., Birney, E., and Furlong, E. E. (2012). A transcription factor collective defines cardiac cell fate and reflects lineage history. Cell 148, 473-486.

Keleman, K., and Dickson, B. J. (2001). Short- and long-range repulsion by the Drosophila Unc5 netrin receptor. Neuron 32, 605-617.

Keshishian, H., Broadie, K., Chiba, A., and Bate, M. (1996). The Drosophila neuromuscular junction: a model system for studying synaptic development and function. Annu. Rev. Neurosci. 19, 545-575.

Klassen, M. P., and Shen, K. (2007). Wnt signaling positions neuromuscular connectivity by inhibiting synapse formation in C. elegans. Cell 130, 704-716.

Kohsaka, H., and Nose, A. (2009). Target recognition at the tips of postsynaptic filopodia: accumulation and function of Capricious. Development 136, 1127-1135.

Kohsaka, H., Takasu, E., and Nose, A. (2007). In vivo induction of postsynaptic molecular assembly by the cell adhesion molecule Fasciclin2. J. Cell Biol. 179, 1289-1300.

Kolodkin, A. L., and Tessier-Lavigne, M. (2011). Mechanisms and molecules of neuronal wiring: a primer. Cold Spring Harb. Perspect. Biol. 3, 1-13.

Kolodziej, P. A., Timpe, L. C., Mitchell, K. J., Fried, S. R., Goodman, C. S., Jan, L. Y., and Jan, Y. N. (1996). Frazzled encodes a Drosophila member of the DCC immunoglobulin subfamily and is required for CNS and motor axon guidance. Cell 87, 197-204.

Kopczynski, C. C., Davis, G. W., and Goodman, C. S. (1996).
A neural tetraspanin, encoded by late bloomer, that facilitates synapse formation. Science 271, 1867-1870.

Kose, H., Rose, D., Zhu, X. M., and Chiba, A. (1997). Homophilic synaptic target recognition mediated by immunoglobulin-like cell adhesion molecule Fasciclin III. Development 124, 4143-4152.

Kurusu, M., Cording, A., Taniguchi, M., Menon, K., Suzuki, E., and Zinn, K. (2008). A screen of cellsurface molecules identifies leucinerich repeat proteins as key mediators of synaptic target selection. Neuron 59, 972-985.

Labrador, J. P., O’Keefe, D., Yoshikawa, S., McKinnon, R. D., Thomas, J. B., and Bashaw, G. J. (2005). The homeobox transcription factor even-skipped regulates netrinreceptor expression to control dorsal motor-axon projections in Drosophila. Curr. Biol. 15, 1413-1419.

Landgraf, M., and Thor, S. (2006). Development and structure of motoneurons. Int. Rev. Neurobiol. 75, 33-53.

Linhoff, M. W., Laurén, J., Cassidy, R. M., Dobie, F. A., Takahashi, H. Nygaard, H. B., Airaksinen, M. S., Strittmatter, S. M., and Craig, A. M. (2009). An unbiased expression screen for synaptogenic proteins identifies the LRRTM protein family as synaptic organizers. Neuron 61, 734-749.

Lohmann, C., and Bonhoeffer, T. (2008). A role for local calcium signaling in rapid synaptic partner selection by dendritic filopodia. Neuron 59, 253-260.

Maeder, C., and Shen, K. (2011). Genetic dissection of synaptic specificity. Curr. Opin. Neurobiol. 21, 93-99.

Matthes, D. J., Sink, H., Kolodkin, A L., and Goodman, C. S. (1995). Semaphorin-II can function as a selective inhibitor of specific synaptic arborizations. Cell 81, 631-639.

Mattila, P. K., and Lappalainen, P. (2008). Filopodia: molecular architecture and cellular functions. Nat. Rev. Mol. Cell Biol. 9, 446-454.

Mitchell, K. J., Doyle, J. L., Serafini, T., Kennedy, T. E., TessierLavigne, M., Goodman, C. S., and Dickson, B. J. (1996). Genetic analysis of Netrin genes in Drosophila: netrins guide CNS commissural axons and peripheral motor axons. Neuron 17 , 203-215.

Morey, M., Yee, S. K., Herman, T., Nern, A., Blanco, E., and Zipursky,
S. L. (2008). Coordinate control of synaptic-layer specificity and rhodopsins in photoreceptor neurons. Nature 456, 795-799.

Mosca, T. J., Hong, W., Dani, V. S., Favaloro, V., and Luo, L. (2012). Trans-synaptic Teneurin signalling in neuromuscular synapse organization and target choice. Nature 484, 237-241.

Nose, A., Mahajan, V. B., and Goodman, C. S. (1992). Connectin: a homophilic cell-adhesion molecule expressed on a subset of muscles and the motoneurons that innervate them in Drosophila. Cell 70, 553-567.

Nose, A., Takeichi, M., and Goodman, C. S. (1994). Ectopic expression of connectin reveals a repulsive function during growth cone guidance and synapse formation. Neuron 13 525-539.

Nose, A., Umeda, T., and Takeichi, M. (1997). Neuromuscular target recognition by a homophilic interaction of Connectin cell adhesion molecules in Drosophila. Development 124, 1433-1441.

Ritzenthaler, S., and Chiba, A. (2003) Myopodia (postsynaptic filopodia) participate in synaptic target recognition. J. Neurobiol. 55, 31-40.

Ritzenthaler, S., Suzuki, E., and Chiba, A. (2000). Postsynaptic filopodia in muscle cells interact with innervating motoneuron axons. Nat. Neurosci. 3, 1012-1017.

Rose, D., and Chiba, A. (1999). A single growth cone is capable of integrating simultaneously presented and functionally distinct molecular cues during target recognition. J. Neurosci. 19, 4899-4906.

Rose, D., and Chiba, A. (2000) Synaptic target recognition at Drosophila neuromuscular junctions. Microsc. Res. Tech. 49, 3-13.

Rose, D., Zhu, X. M., Kose, H., Hoang B., Cho, J., and Chiba, A. (1997) Toll, a muscle cell surface molecule, locally inhibits synaptic initiation of the RP3 motoneuron growth cone in Drosophila. Development 124 , 1561-1571.

Roy, S., Hsiung, F., and Kornberg, T. B. (2011). Specificity of Drosophila cytonemes for distinct signaling pathways. Science 332 , 354-358.

Ruiz-Canada, C., and Budnik, V. (2006). Introduction on the use of the Drosophila embryonic/larval neuromuscular junction as a model system to study synapse development and function, and a brief summary of pathfinding and target recognition. Int. Rev. Neurobiol. 75, $1-31$

Rørth, P. (2003). Communication by touch: role of cellular extensions in complex animals. Cell 112, 595-598.

Sanes, J. R., and Yamagata, M. (2009). Many paths to synaptic specificity. Annu. Rev. Cell Dev. Biol. 25, 161-195.

Shen, K., and Scheiffele, P. (2010). Genetics and cell biology of building specific synaptic connectivity. Annu. Rev. Neurosci. 33, 473-507.

Shinza-Kameda, M., Takasu, E., Sakurai, K., Hayashi, S., and Nose, A. (2006). Regulation of layer-specific targeting by reciprocal expression of a cell adhesion molecule, capricious. Neuron 49, 205-213.

Shishido, E., Takeichi, M., and Nose, A. (1998). Drosophila synapse formation: regulation by transmembrane protein with leu-rich repeats, CAPRICIOUS. Science 280 , 2118-2121.

Siebert, M., Banovic, D., Goellner, B., and Aberle, H. (2009). Drosophila motor axons recognize and follow a sidestep-labeled substrate pathway to reach their target fields. Genes Dev. 23, 1052-1062

Sink, H., and Whitington, P. M. (1991a). Location and connectivity of abdominal motoneurons in the embryo and larva of Drosophila melanogaster. J. Neurobiol. 22, 298-311.

Sink, H., and Whitington, P. M. (1991b). Pathfinding in the centralnervous-system and periphery by identified embryonic Drosophila motor axons. Development 112, 307-316.

Sink, H., and Whitington, P. M. (1991c). Early ablation of target muscles modulates the arborisation pattern of an identified embryonic Drosophila motor axon. Development 113, 701-707.

Sperry, R. W. (1963). Chemoaffinity in the orderly growth of nerve fiber patterns and connections. Proc. Natl. Acad. Sci. U.S.A. 50, 703-710.

Taniguchi, H., Shishido, E., Takeichi, M., and Nose, A. (2000). Functional dissection of Drosophila Capricious: its novel roles in neuronal pathfinding and selective synapse formation. J. Neurobiol. 42, 104-116.

Tixier, V., Bataille, L., and Jagla, K. (2010). Diversification of muscle types: recent insights from Drosophila. Exp. Cell Res. 316, 3019-3027.

Umemiya, T., Takasu, E., Takeichi, M., Aigaki, T., and Nose, A 
(2002). Forked end: a novel transmembrane protein involved in neuromuscular specificity in Drosophila identified by gain-offunction screening. J. Neurobiol. 51, 205-214.

Valanne, S., Wang, J. H., and Rämet, M. (2011). The Drosophila toll signaling pathway. J. Immunol. 186, 649-656.

Van Vactor, D., Sink, H., Fambrough, D., Tsoo, R., and Goodman, C. S. (1993). Genes that control neuromuscular specificity in Drosophila. Cell 73, 1137-1153.
West, A. P., Koblansky, A. A., and Ghosh, S. (2006). Recognition and signaling by toll-like receptors. Annu. Rev. Cell Dev. Biol. 22, 409-437.

Winberg, M. L., Mitchell, K. J., and Goodman, G. S. (1998). Genetic analysis of the mechanisms controlling target selection: complementary and combinatorial functions of netrins, semaphorins, and IgCAMs. Cell 93, 581-591.

Zinzen, R. P., Girardot, C., Gagneur, J., Braun, M., and Furlong, E. E. (2009). Combinatorial binding predicts spatio-temporal cisregulatory activity. Nature 462, 65-70.

Conflict of Interest Statement: The author declares that the research was conducted in the absence of any commercial or financial relationships that could be construed as a potential conflict of interest.

Received: 08 March 2012; paper pending published: 28 March 2012; accepted:
23 April 2012; published online: 08 May 2012.

Citation: Nose A (2012) Generation of neuromuscular specificity in Drosophila: novel mechanisms revealed by new technologies. Front. Mol. Neurosci. 5:62. doi: 10.3389/fnmol.2012.00062

Copyright (c) 2012 Nose. This is an open-access article distributed under the terms of the Creative Commons Attribution Non Commercial License, which permits non-commercial use, distribution, and reproduction in other forums, provided the original authors and source are credited. 